\title{
Responsabilidad por pérdida de oportunidad asistencial en la medicina pública española.
}

\author{
Sardinero García, Carlos, Santiago Sáez, Andrés, Perea \\ Pérez, Bernardo, Albarrán Juan, María Elena, Labajo \\ González, Elena y Benito León, Julián.
}

Cita:

Sardinero García, Carlos, Santiago Sáez, Andrés, Perea Pérez, Bernardo, Albarrán Juan, María Elena, Labajo González, Elena y Benito León, Julián (2017). Responsabilidad por pérdida de oportunidad asistencial en la medicina pública española. Revista Española de Medicina Legal, 43, 5-12.

Dirección estable: https://www.aacademica.org/elenalabajogonzalez/31 ARK: https://n2t.net/ark:/13683/pcQr/tKX

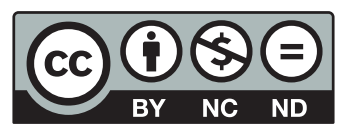




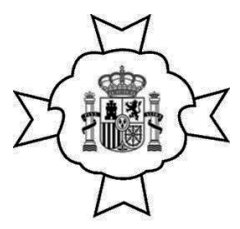

ASOCLACIÓN NACIONAL MÉDICOS FORENSES
REVISTA ESPAÑOLA DE

MEDICINA LEGAL

ORIGINAL

\title{
Responsabilidad por pérdida de oportunidad asistencial en la medicina pública española
}

\section{Carlos Sardinero-García ${ }^{\mathrm{a}, \mathrm{b}}$, Andrés Santiago-Sáez $^{\mathrm{c}, \mathrm{d}}$, Bernardo Perea-Pérez ${ }^{\mathrm{d}}$, M. Elena Albarrán-Juan ${ }^{\mathrm{d}}$, Elena Labajo-González ${ }^{\mathrm{d}}$ y Julián Benito-León ${ }^{\mathrm{e}, \mathrm{f}, \mathrm{g}, *}$}

\author{
a Sardinero Abogados, Leganés, Madrid \\ b Departamento de Derecho Penal, Procesal e Historia del Derecho, Facultad de Ciencias Sociales y Jurídicas, \\ Universidad Carlos III, Getafe, Madrid, España \\ c Servicio de Medicina Legal, Hospital Universitario Clínico San Carlos, Madrid, España \\ d Departamento de Toxicología y Legislación Sanitaria, Escuela de Medicina Legal, Facultad de Medicina, \\ Universidad Complutense, Madrid, España \\ e Servicio de Neurología, Hospital Universitario 12 de Octubre, Madrid, España \\ f Departamento de Medicina, Facultad de Medicina, Universidad Complutense, Madrid, España \\ s Centro de Investigación Biomédica en Red sobre Enfermedades Neurodegenerativas, Madrid, España
}

Recibido el 11 de abril de 2016; aceptado el 28 de junio de 2016

Disponible en Internet el 31 de agosto de 2016

\section{PALABRAS CLAVE \\ Asistencia sanitaria; \\ Condenas; \\ Indemnización; \\ Pérdida de \\ oportunidad}

\begin{abstract}
Resumen
Introducción: La pérdida de oportunidad asistencial es un concepto jurídico que se ha abierto paso en los últimos años como un criterio determinante para la valoración del daño corporal derivado de la asistencia sanitaria. En este trabajo analizamos las sentencias condenatorias dictadas por la Jurisdicción Contencioso Administrativa, en el ámbito de la medicina pública española, en los supuestos en los que, por falta de diligencia del profesional, el paciente ha perdido la posibilidad de un diagnóstico o un tratamiento adecuado.

Material y métodos: Se han seleccionado las sentencias judiciales, relacionadas con la medicina pública española, que hacían referencia al concepto de pérdida de oportunidad asistencial y que fueron dictadas hasta mayo del 2014.

Resultados: De las 519 sentencias analizadas, 340 (66,5\%) fueron dictadas por error diagnóstico y $138(26,6 \%)$ por tratamiento inadecuado. Desde el año 2010 se produjeron $351(67,6 \%)$ sentencias, lo que supuso más del $100 \%$ de aumento con respecto a las 168 (32,4\%) ocurridas en los primeros 8 años del estudio (2002 a 2009). La mayoría de los pacientes fueron varones $(56,1 \%)$, que fallecieron $(57,4 \%)$, siendo las patologías que más frecuentemente originaron la condena, la oncológica maligna $(26,4 \%)$ y la neurológica $(17,3 \%)$.
\end{abstract}

\footnotetext{
* Autor para correspondencia.

Correo electrónico: jbenitol67@gmail.com (J. Benito-León).
} 
Conclusiones: La actividad litigante por pérdida de oportunidad asistencial en la medicina pública española ha aumentado significativamente estos últimos años. Las condenas fueron dictadas fundamentalmente por error diagnóstico y tratamiento inadecuado.

(C) 2016 Asociación Nacional de Médicos Forenses. Publicado por Elsevier España, S.L.U. Todos los derechos reservados.

\section{KEYWORDS}

Health care; Convictions; Compensation; Loss of chance

\section{Introducción}

El concepto de pérdida de oportunidad asistencial ha surgido con la intención de ofrecer una solución jurídica a supuestos en los que, por la omisión de la diligencia debida, se ha privado al paciente de una posibilidad de curación ${ }^{1-3}$. Saigí Ullastre et al. ${ }^{4}$ definen el concepto de pérdida de oportunidad como aquellos supuestos que presentan, como característica común, un error o retraso de diagnóstico de un proceso médico que acaba con lesiones o el fallecimiento del paciente. Desde el punto de vista de la responsabilidad médica, toda omisión hace referencia a una acción concreta; la acción que cabía esperar del facultativo que tenía la obligación de actuar y, más bien, de actuar de determinada manera, es decir, con arreglo a la lex artis $^{3}$. En estos supuestos, la omisión provoca que se desconozca con certeza lo que habría sucedido en caso de sustituir tal conducta por la acción que resultaba adecuada, motivo por el que se desconoce si el resultado se ha producido con toda seguridad por la omisión del profesional médico ${ }^{1-3}$. No sabemos si en caso de haber actuado correctamente el daño se habría producido de la misma manera ${ }^{1-3}$.

Efectivamente, nos encontramos ante complejos supuestos de prueba de la relación de causalidad, que no permite conocer y acreditar lo que habría ocurrido si el autor de tal conducta hubiera actuado correctamente ${ }^{1-3}$. Para ello, habría que saber si, al añadir mentalmente la conducta omitida, el resultado lesivo se habría producido de igual forma ${ }^{3}$.
En este artículo pretendemos determinar las características de las sentencias dictadas por pérdida de oportunidad asistencial en el ámbito de la medicina pública española, considerando todas las variables que contemplan alguna influencia, tanto en el planteamiento de la reclamación como en la resolución del proceso judicial. De este modo, los resultados podrían facilitar una práctica más segura de la medicina, tanto para el paciente como para el profesional, así como establecer unos criterios que ofrezcan alguna previsibilidad ante posibles reclamaciones judiciales envueltas por la pérdida de oportunidad. Finalmente, se debe destacar que no existe en la literatura médica o jurídica ningún estudio similar que haga referencia específica a la pérdida de oportunidad asistencial en medicina.

\section{Métodos}

\section{Origen del material y criterios de inclusión}

Con carácter previo, es conveniente aclarar que la jerarquía jurisdiccional española se establece por su función procesal $^{1}$. Así, podemos distinguir, por un lado, los órganos de instancia - primera o única - que son los que resuelven los asuntos por primera vez, y, por otro, la segunda instancia que sirve para realizar una función revisora de la primera; es decir, un nuevo enjuiciamiento pleno, ante un órgano superior estratificado de la siguiente forma en la vía contencioso 
administrativa: Tribunal Superior de Justicia (órgano superior en la comunidad autónoma con competencias para juzgar responsabilidad patrimonial tras la derivación de competencias desde el Estado a las comunidades autónomas), Audiencia Nacional (todavía con competencias en materia de responsabilidad patrimonial derivada de asistencia sanitaria en competencias no transferidas a las comunidades autónomas, como las que ejerce el Ministerio de Sanidad, Servicios Sociales e Igualdad o en competencias exclusivas del Estado, como las que ejerce el Ministerio de Defensa) y; finalmente, el Tribunal Supremo (órgano jurisdiccional superior). En la vía de reclamación contencioso administrativa existe un complejo sistema competencial que contempla la Ley 29/1998, de 13 de julio, reguladora de la Jurisdicción Contencioso Administrativa, en la que dependiendo del ámbito territorial se acude en primera instancia a los juzgados de lo Contencioso-Administrativo o directamente al Tribunal Superior de Justicia de la comunidad autónoma, sin necesidad de acudir antes a los juzgados de lo Contencioso-Administrativo.

Se han analizado todas las sentencias judiciales, relacionadas con patologías médicas, quirúrgicas y maternoinfantiles, en el ámbito de la Jurisdicción Contencioso Administrativa, que hacían referencia al concepto de pérdida de oportunidad asistencial y que fueron dictadas en España hasta mayo de 2014. Se hizo la búsqueda, durante el mes de mayo del 2014, utilizando la base de datos jurídica de la editorial El Derecho (http: / / www.elderecho.com/) por considerarla, en la actualidad, como una de las bases más potentes y más utilizadas, tanto en el ámbito académico, como en el profesional, por letrados, jueces y magistrados.

En la base de recogida de datos se recogieron las variables administrativas, judiciales y clínicas de cada sentencia. No se pudo recoger la variable «tipo de profesional implicado» debido a que al ser sentencias en el ámbito contencioso administrativo no se especificaba, en la mayor parte de ellas, qué médico (especialista o no) había participado. La vía de acceso (motor de búsqueda) fue la siguiente: responsabilidad patrimonial/asistencia sanitaria/pérdida de oportunidad/condena.

\section{Análisis estadístico}

Se realizó un estudio descriptivo simple de las variables recogidas, mediante porcentajes. En lo referente a las comunidades autónomas, con objeto de corregir el número de sentencias respecto a la actividad médica realizada, se ha presentado el número de camas hospitalarias públicas, según datos del Ministerio de Sanidad, Servicios Sociales e Igualdad actualizado a 31 de diciembre de 2014 (http://www.msssi.gob.es/ciudadanos/prestaciones/ centrosServiciosSNS/hospitales/docs/CNH2015.pdf). De esta manera, obtuvimos el índice de sentencias por cada 100.000 camas hospitalarias. En los conjuntos de 2 variables cuya distribución pudiera estar relacionada y esa relación pudiera ser de interés se ha realizado un contraste de variables mediante tablas de contingencia usando la prueba de la chi al cuadrado o el test exacto de Fisher. Para comparar la cuantía indemnizatoria respecto a la solicitada se empleó la prueba de los rangos con signos de Wilcoxon. La significación estadística se estableció para un valor
Tabla 1 Variables administrativas

\begin{tabular}{|c|c|}
\hline Variables & N (\%) \\
\hline \multicolumn{2}{|l|}{ Fecha de la sentencia } \\
\hline 2002-2009 & $168(32,4)$ \\
\hline $2010-2014$ & $351(67,6)$ \\
\hline \multicolumn{2}{|c|}{$\begin{array}{l}\text { Comunidad Autónoma (número de camas hospitalarias } \\
\text { públicas) }^{\text {a }}\end{array}$} \\
\hline Andalucía (15.391) & $24(4,6)[155,9]$ \\
\hline Aragón (4.527) & $30(5,8)[662,7]$ \\
\hline Canarias (4.799) & $5(1,0)[104,2]$ \\
\hline Cantabria (1.366) & $10(1,9)[732,1]$ \\
\hline Castilla-La Mancha (5.300) & $14(2,7)[264,1]$ \\
\hline Castilla y León (7.347) & $69(13,3)[939,1]$ \\
\hline Extremadura (2.977) & $10(1,9)[369,3]$ \\
\hline Galicia (7.311) & $27(5,2)[369,3]$ \\
\hline Illes Balears (2.581) & $4(0,8)[155,0]$ \\
\hline La Rioja (808) & $5(1,0)[618,8]$ \\
\hline $\begin{array}{l}\text { Comunidad de Madrid } \\
\text { (14.853) }\end{array}$ & $134(25,8)[902,2]$ \\
\hline Región de Murcia (3.191) & $14(2,7)[438,7]$ \\
\hline $\begin{array}{l}\text { Comunidad Valenciana } \\
\text { (11.441) }\end{array}$ & $68(13,1)[594,3]$ \\
\hline $\begin{array}{l}\text { Principado de Asturias } \\
\text { (2.761) }\end{array}$ & $30(5,8)[1.086,6]$ \\
\hline Cataluña (14.795) & $9(1,7)[60,8]$ \\
\hline $\begin{array}{l}\text { Comunidad Foral de Navarra } \\
(1.405)\end{array}$ & $1(0,2)[71,2]$ \\
\hline País Vasco $(5.808)$ & $3(0,6)[51,6]$ \\
\hline
\end{tabular}

Datos presentados como $\mathrm{N}$ (\%) [índice de sentencias/100.000 camas hospitalarias].

a Treinta y tres $(6,4 \%)$ casos se resolvieron en casación en el Tribunal Supremo y $29(5,6 \%)$ en primera instancia en la Audiencia Nacional; el resto se resolvió en los correspondientes Tribunales Superiores de Justicia de cada comunidad.

Número de camas hospitalarias públicas: 107.083.

$p<0,05$. Los datos se analizaron mediante el programa estadístico SPSS v. 21.0.

\section{Resultados}

De las 10.001 sentencias obtenidas, 1.249 aludían al concepto de pérdida de oportunidad asistencial. De estas, 522 fueron condenas en el ámbito de la medicina pública; decidimos excluir 3 de ellas por no figurar la patología o secuelas que produjeron la reclamación. Dichas condenas fueron dictadas desde el 16 de octubre del 2002 hasta el 14 de mayo del 2014.

La primera sentencia de nuestra muestra por pérdida de oportunidad asistencial en España, en el ámbito de la medicina pública (no detección en la ecografía de una focomelia durante el embarazo), se produjo el 16 de octubre del 2002 por la Audiencia Nacional. Desde entonces, se ha producido un crecimiento exponencial de este tipo de sentencias (tabla 1). Así, desde el año 2010 se han encontrado 351 (67,6\%) sentencias, lo que supone más del $100 \%$ de aumento con respecto a las $168(32,4 \%)$ sentencias ocurridas en los primeros 8 años del estudio (2002 a 2009). De las 17 comunidades autónomas, las de Madrid, Castilla y León, y Valencia fueron, 
Tabla 2 Variables clínicas

\begin{tabular}{|c|c|c|}
\hline Variables & Categorías & $\mathrm{N}(\%)$ \\
\hline \multicolumn{3}{|l|}{ Edad $^{\mathrm{a}}$} \\
\hline & $\leq 14$ años & $58(11,2)$ \\
\hline & 15-64 años & $170(32,8)$ \\
\hline & $\geq 65$ años & $100(19,3)$ \\
\hline \multicolumn{3}{|l|}{ Sexo } \\
\hline & Hombre & $291(56,1)$ \\
\hline & Mujer & $228(43,9)$ \\
\hline \multicolumn{3}{|c|}{ Patología que llevó a la reclamación } \\
\hline & $\begin{array}{l}\text { Patología oncológica } \\
\text { maligna (excluyendo los } \\
\text { tumores del sistema } \\
\text { nervioso central) }\end{array}$ & $137(26,4)$ \\
\hline & $\begin{array}{l}\text { Enfermedades } \\
\text { neurológicas }\end{array}$ & $90(17,3)$ \\
\hline & Cardiopatías & $78(15,0)$ \\
\hline & $\begin{array}{l}\text { Enfermedades } \\
\text { infecciosas }\end{array}$ & $48(9,2)$ \\
\hline & $\begin{array}{l}\text { Enfermedades del } \\
\text { aparato digestivo }\end{array}$ & $43(8,3)$ \\
\hline & Patología traumatológica & $38(7,3)$ \\
\hline & $\begin{array}{l}\text { Patologías } \\
\text { oftalmológicas }\end{array}$ & $22(4,2)$ \\
\hline & $\begin{array}{l}\text { Malformaciones fetales } \\
\text { no detectadas }\end{array}$ & $11(2,1)$ \\
\hline & Miscelánea & $52(10,0)$ \\
\hline \multicolumn{3}{|c|}{ Patología recodificada } \\
\hline & Médica & $296(57,0)$ \\
\hline & Quirúrgica & $152(29,3)$ \\
\hline & Materno-Infantil & $71(13,7)$ \\
\hline
\end{tabular}

${ }^{a}$ En 191 (36,8\%) casos no consta la edad en la sentencia.

en valor absoluto, donde se dictaron más sentencias condenatorias por pérdida de oportunidad; sin embargo, tras la «corrección» por el número de camas, el índice de sentencias por cada 100.000 camas hospitalarias fue máximo en el Principado de Asturias y en la Castilla y León (tabla 1).

En la tabla 2 se muestran las variables clínicas. La mayoría de los pacientes fueron varones $(56,1 \%)$, siendo las patologías que más frecuentemente originaron la condena la patología oncológica maligna $(26,4 \%)$ y la neurológica $(17,3 \%)$.

En la tabla 3 se presentan las variables judiciales. La mayor parte de las condenas fueron impuestas a la comunidad autónoma y a su aseguradora (compañía de seguros) $(88,2 \%)$. De las 519 sentencias, 340 (66,5\%) fueron dictadas por error diagnóstico (ya sea por no solicitar una prueba en concreto o por una exploración deficiente), $138(26,6 \%)$ por la omisión de un tratamiento adecuado y tan solo 18 (3,5\%) por omisión de información. Un 57,4\% de los pacientes falleció. Es de destacar que la distribución de la cuantía inicialmente reclamada es inferior a la de la indemnización finalmente concedida. Si bien son mayoritarias las reclamaciones comprendidas entre los 100.000 y los 499.999 euros $(54,3 \%)$, los resultados reflejan que son mayoritarias $(80 ., 5 \%)$ las indemnizaciones concedidas $\leq 100.000$ euros. En general, la cuantía
Tabla 3 Variables judiciales

\begin{tabular}{|c|c|c|}
\hline Variables & Categorías & $\mathrm{N}(\%)$ \\
\hline \multirow[t]{2}{*}{$\begin{array}{l}\text { Entidades } \\
\text { demandadas }\end{array}$} & $\begin{array}{l}\text { Comunidad autónoma y } \\
\text { aseguradora }\end{array}$ & $458(88,2)$ \\
\hline & $\begin{array}{l}\text { Solo comunidad } \\
\text { autónoma }\end{array}$ & $61(11,8)$ \\
\hline \multirow{4}{*}{$\begin{array}{l}\text { Hecho imputado } \\
\text { motivo de la } \\
\text { sentencia }\end{array}$} & Error diagnóstico & $340(66,5)$ \\
\hline & Tratamiento inadecuado & $138(26,6)$ \\
\hline & $\begin{array}{l}\text { Omisión de } \\
\text { consentimiento } \\
\text { informado }\end{array}$ & $18(3,5)$ \\
\hline & $\begin{array}{l}\text { Mezcla de distintos } \\
\text { hechos imputados }\end{array}$ & $23(4,4)$ \\
\hline \multirow{2}{*}{$\begin{array}{l}\text { Resultado del } \\
\text { daño demandado }\end{array}$} & Fallecimiento & $298(57,4)$ \\
\hline & Lesiones/secuelas & $218(42,0)$ \\
\hline \multirow{3}{*}{$\begin{array}{l}\text { Cuantía } \\
\text { económica de la } \\
\text { reclamación }{ }^{\mathrm{b}}\end{array}$} & $\leq 100.000$ euros & $90(17,3)$ \\
\hline & $100.001-499.999$ euros & $282(54,3)$ \\
\hline & $\geq 500.000$ & $52(10,0)$ \\
\hline \multirow{3}{*}{$\begin{array}{l}\text { Cuantía de la } \\
\text { indemnización }\end{array}$} & $\leq 100.000$ euros & $418(80,5)$ \\
\hline & 100.001-499.999 euros & $97(18,7)$ \\
\hline & $\geq 500.000$ euros & $4(0,8)$ \\
\hline \multirow{3}{*}{$\begin{array}{l}\text { Modo de } \\
\text { aplicación de la } \\
\text { cuantía }\end{array}$} & Tanto alzado & $426(82,1)$ \\
\hline & Baremo de tráfico & $49(9,4)$ \\
\hline & $\begin{array}{l}\text { Porcentaje de } \\
\text { posibilidades }\end{array}$ & $44(8,5)$ \\
\hline
\end{tabular}

a En $3(0,6 \%)$ casos no se especificó en la sentencia el resultado del daño reclamado.

b En $95(18,3 \%)$ casos no se especificó en la sentencia la indemnización reclamada (se supone que fue por cuantía indeterminada).

concedida (mediana $=50.000$ euros) fue inferior a la solicitada (mediana $=180.301 .5$ euros) (prueba de los rangos con signos de Wilcoxon, $p<0,001$ ). No encontramos diferencias estadísticamente significativas (U de Mann-Whitney, $p=0,09$ ) entre las indemnizaciones concedidas en el primer periodo del estudio (2004-2009) (mediana $=60.000$ euros) $\mathrm{y}$ el segundo (2010-2014) (mediana $=40.000$ euros). La cuantía mayor (casi un millón de euros) se dictó en el caso de un varón en el que no se especificaba la edad a la que había presentado un ictus y en el que se había dado un error diagnóstico. Finalmente, resulta muy relevante que solo un $9,4 \%$ se aplique por analogía o identidad de razón el baremo de tráfico, en un $8,5 \%$ se indemnice en atención al porcentaje hipotético de posibilidades de curación (porcentaje que se aplica sobre el citado baremo) y que, en la mayoría de los casos $(82,1 \%)$, se indemnice a tanto alzado, es decir, sin atender al baremo ni a criterios delimitados (tabla 3).

La tabla 4 muestra que la proporción de indemnizaciones mayores ( $\geq 500.000$ euros) fueron concedidas a pacientes en edad pediátrica y en casos de malformaciones fetales no detectadas. Sobre la importancia de la patología materno-filial, en pérdida de oportunidad, ya existe algún trabajo previo realizado en España ${ }^{5}$. Asimismo, cuando se recodificaron las patologías en médicas, quirúrgicas y materno-infantiles, fueron estas últimas las que más indemnización proporcionalmente recibieron (tabla 4). Las secuelas o lesiones recibieron proporcionalmente más indemnizaciones que si se había producido un fallecimiento 
Tabla 4 Desglose de la indemnización final de acuerdo con las variables clínicas, administrativas y judiciales

\begin{tabular}{|c|c|c|c|c|}
\hline & \multicolumn{3}{|c|}{ Indemnización final } & \multirow[t]{2}{*}{ Valor $\mathrm{p}^{\mathrm{b}}$} \\
\hline & $\leq 100.000$ euros & 100.001-499.999 euros & $\geq 500.000$ euros & \\
\hline Edad $^{\mathrm{a}}$ & & & & $<0,0001$ \\
\hline$\leq 14$ años & $31(53,4 \%)$ & $24(41,4 \%)$ & $3(5,2 \%)$ & \\
\hline 15-64 años & $135(79,4 \%)$ & $35(20,6 \%)$ & $0(0,0 \%)$ & \\
\hline$\geq 65$ años & $89(89,0 \%)$ & $11(11,0 \%)$ & $0(0,0 \%)$ & \\
\hline Sexo & & & & 0,436 \\
\hline Hombre & $229(78,7 \%)$ & $60(20,6 \%)$ & $2(0,7 \%)$ & \\
\hline Mujer & $189(82,9 \%)$ & $37(16,2 \%)$ & $2(0,9 \%)$ & \\
\hline Patología que originó la reclamación & & & & $<0,0001$ \\
\hline Patología oncológica maligna & $114(83,2 \%)$ & $23(16,8 \%)$ & $0(0,0 \%)$ & \\
\hline Enfermedades neurológicas & $62(68,9 \%)$ & $24(26,7 \%)$ & $4(4,4 \%)$ & \\
\hline Cardiopatías & $63(80,8 \%)$ & $15(19,2 \%)$ & $0(0,0 \%)$ & \\
\hline Enfermedades infecciosas & $41(85,4 \%)$ & $7(14,6 \%)$ & $0(0,0 \%)$ & \\
\hline Enfermedades del aparato digestivo & $39(90,7 \%)$ & $4(9,3 \%)$ & $0(0,0 \%)$ & \\
\hline Patología traumatológica & $32(84,2 \%)$ & $6(15,8 \%)$ & $0(0,0 \%)$ & \\
\hline Patologías oftalmológicas & $21(95,5 \%)$ & $1(4,5 \%)$ & $0(0,0 \%)$ & \\
\hline Malformaciones fetales no detectadas & $2(18,2 \%)$ & $9(81,8 \%)$ & $0(0,0 \%)$ & \\
\hline Miscelánea & $44(84,6 \%)$ & $8(15,4 \%)$ & $0(0,0 \%)$ & \\
\hline Patología recodificada & & & & 0,001 \\
\hline Médica & $240(81,1 \%)$ & $54(18,2 \%)$ & $2(0,7 \%)$ & \\
\hline Quirúrgica & $133(87,5 \%)$ & $18(11,8 \%)$ & $1(0,7 \%)$ & \\
\hline Materno-Infantil & $45(63,4 \%)$ & $25(35,2 \%)$ & $1(1,4 \%)$ & \\
\hline Periodo de la reclamación & & & & 0,380 \\
\hline $2002-2009$ & $136(81,0 \%)$ & $32(19,0 \%)$ & $0(0,0 \%)$ & \\
\hline $2010-2014$ & $282(80,3 \%)$ & $65(18,5 \%)$ & $4(1,1 \%)$ & \\
\hline Entidades demandadas & & & & 0,753 \\
\hline Comunidad autónoma y aseguradora & $368(80,3 \%)$ & $86(18,8 \%)$ & $4(0,9 \%)$ & \\
\hline Solo comunidad autónoma & $50(82,0 \%)$ & $11(18,0 \%)$ & $0(0,0 \%)$ & \\
\hline Hecho imputado motivo de la sentencia & & & & 0,334 \\
\hline Error diagnóstico & $272(80,0 \%)$ & $67(19,4 \%)$ & $2(0,6 \%)$ & \\
\hline Tratamiento inadecuado & $111(80,4 \%)$ & $26(18,8 \%)$ & $1(0,7 \%)$ & \\
\hline Omisión de consentimiento informado & $15(83,3 \%)$ & $2(11,1 \%)$ & $1(5,6 \%)$ & \\
\hline Mezcla de distintos hechos imputados & $20(87,0 \%)$ & $3(13,0 \%)$ & $0(0,0 \%)$ & \\
\hline Resultado del daño demandado & & & & 0,043 \\
\hline Fallecimiento & $246(82,6 \%)$ & $52(17,4 \%)$ & $0(0,0 \%)$ & \\
\hline Lesiones/secuelas & $170(78,0 \%)$ & $44(20,2 \%)$ & $4(1,8 \%)$ & \\
\hline Modo de aplicación de la cuantía & & & & 0,001 \\
\hline Tanto alzado & $355(83,3 \%)$ & $69(16,2 \%)$ & $2(0,5 \%)$ & \\
\hline Baremo de tráfico & $30(61,2 \%)$ & $17(34,7 \%)$ & $2(4,1 \%)$ & \\
\hline Porcentaje de posibilidades & $33(75,0 \%)$ & $11(25,0 \%)$ & $0(0,0 \%)$ & \\
\hline Comunidad autónoma & & & & $<0,0001$ \\
\hline Andalucía & $17(70,8 \%)$ & $7(29,2 \%)$ & $0(0,0 \%)$ & \\
\hline Aragón & $27(90,0 \%)$ & $3(10,0 \%)$ & $0(0,0 \%)$ & \\
\hline Canarias & $3(60,0 \%)$ & $2(40,0 \%)$ & $0(0,0 \%)$ & \\
\hline Cantabria & $6(60,0 \%)$ & $4(40,0 \%)$ & $0(0,0 \%)$ & \\
\hline Castilla-La Mancha & $10(71,4 \%)$ & $4(28,6 \%)$ & $0(0,0 \%)$ & \\
\hline Castilla y León & $63(91,3 \%)$ & $6(8,7 \%)$ & $0(0,0 \%)$ & \\
\hline Extremadura & $7(70,0 \%)$ & $3(30,0 \%)$ & $0(0,0 \%)$ & \\
\hline Galicia & $23(85,2 \%)$ & $4(14,8 \%)$ & $0(0,0 \%)$ & \\
\hline Illes Balears & $4(100,0 \%)$ & $0(0,0 \%)$ & $0(0,0 \%)$ & \\
\hline La Rioja & $5(100,0 \%)$ & $0(0,0 \%)$ & $0(0,0 \%)$ & \\
\hline Comunidad de Madrid & $99(73,9 \%)$ & $34(25,4 \%)$ & $1(0,7 \%)$ & \\
\hline
\end{tabular}


Tabla 4 (continuación)

\begin{tabular}{llll}
\hline & & Indemnización final & \\
\cline { 2 - 4 } & $\leq 100.000$ euros & $100.001-499.999$ euros & $\geq 500.000$ euros \\
\hline Región de Murcia & $10(71,4 \%)$ & $4(28,6 \%)$ & $0(0,0 \%)$ \\
Comunidad Valenciana & $67(98,5 \%)$ & $1(1,5 \%)$ & $0(0,0 \%)$ \\
Principado de Asturias & $28(93,3 \%)$ & $2(6,7 \%)$ & $0(0,0 \%)$ \\
Cataluña & $6(66,7 \%)$ & $3(33,3 \%)$ & $0(0,0 \%)$ \\
Comunidad foral de Navarra & $1(100,0 \%)$ & $0(0,0 \%)$ & $0(0,0 \%)$ \\
País vasco & $3(100,0 \%)$ & $0(0,0 \%)$ & $0(0,0 \%)$ \\
\hline a En 191 (36,8\%) casos no consta la edad en la sentencia. & & \\
b Chi al cuadrado o test exacto de Fisher. & &
\end{tabular}

(tabla 4). Finalmente, en las comunidades autónomas de Canarias y Cantabria se concedieron las mayores indemnizaciones (tabla 4).

\section{Discusión}

Nuestra investigación ha partido del estudio de sentencias emitidas en supuestos de responsabilidad patrimonial de la administración pública. De esta forma, hemos descartado todas las resoluciones de la jurisdicción civil y penal, para así evitar que nuestros resultados quedasen «contaminados», dado que los criterios de imputación resultan diferentes en atención a la jurisdicción que juzga los actos médicos. En la mayor parte de trabajos españoles se han llevado a cabo sin separar los 3 tipos de jurisdicciones (civil, penal y administrativa), lo que hace difícil comparar los resultados con el presente estudio ${ }^{6-11}$.

La descripción de las sentencias dictadas por pérdida de oportunidad asistencial en la medicina pública española, a lo largo de 12 años, nos muestra principalmente que han experimentado más del $100 \%$ de aumento en los últimos años. Fue a partir del 2002 cuando esta teoría se introdujo con intensidad en la doctrina jurisprudencial a la hora de juzgar la conducta de los profesionales de la medicina, razón por la que podemos sostener que se trata de una teoría con pocos años de aplicación, pero que con el paso del tiempo ha cobrado más presencia.

Nuestros datos revelan distintas cuestiones que es preciso destacar: No todas las comunidades aplican la teoría de la pérdida de oportunidad con la misma frecuencia; las cuantías indemnizatorias también varían en atención a la zona geográfica; tanto la patología oncológica maligna, como la neurológica, fueron las que más frecuentemente originaron la condena, y, finalmente, el error de diagnóstico fue el motivo de condena más repetido.

Los resultados reflejan que, en proporción, y a pesar de no contar con el mayor número de sentencias dictadas, son el Principado de Asturias y Castilla y León las comunidades que más han aplicado la pérdida de oportunidad asistencial, pero, en realidad, su efecto se extiende con vigor a lo largo del panorama nacional en el que Madrid y Valencia también resultaron protagonistas.

En relación con las cuantías indemnizatorias, estas fueron mayoritariamente $(80,5 \%)$ iguales o inferiores a los
100.000 euros, a pesar de que el motivo más frecuente de las reclamaciones fue el fallecimiento, derivado de un error diagnóstico o de un tratamiento inadecuado. Ello refleja una discrepancia con la cuantía reclamada, que solo fue igual o inferior a los 100.000 euros en un $17,3 \%$ de los casos. Los resultados citados tienen una explicación. Cuando un médico omite la aplicación de un tratamiento - p. ej., ante un infarto agudo de miocardio con elevación del segmento ST y bien diagnosticado no indica una rápida restauración mecánica del flujo coronario mediante un stent- y el paciente fallece o presenta secuelas incapacitantes, nunca sabremos si habría sucedido lo mismo de haber aplicado el tratamiento apropiado y a tiempo. En tal supuesto, nadie podría afirmar que el paciente habría sobrevivido sin secuelas. Por esta razón, la doctrina jurisprudencial encuentra enormes dificultades a la hora de valorar si hay responsabilidad y, en su caso, la determinación de la indemnización que merece el resultado dañoso. Lógicamente, ya que solo se puede hablar, como mucho, de una relación de causalidad hipotética, observamos que las condenas reducen significativamente la cantidad reclamada, porque parece que los juzgadores no quieren responsabilizar de todo el daño al facultativo. Y para resolver este debate no hay consenso en la jurisprudencia, pero debemos destacar que en 426 casos $(82,1 \%)$ la indemnización se concedió a tanto alzado, en concepto de daño moral y sin que se sepa a qué criterio concreto acudieron, lo que también provocó una reducción de la indemnización con relación a la cuantía reclamada y a lo que establece el baremo. En 49 casos $(9,4 \%)$ se indemnizó íntegramente el daño con la aplicación del baremo pensado para la valoración del daño corporal en accidentes de tráfico, al no existir un baremo por lesiones de origen sanitario, y en 44 supuestos (8.5\%) se redujo la indemnización en atención al porcentaje de posibilidades de que el daño no se hubiera producido con una actuación diligente.

El primer criterio es precisamente el seguido por la Sección 10. a del Tribunal Superior de Justicia de Madrid, que ha llegado a indemnizar de forma habitual con el importe de 12.000 a 18.000 euros en supuestos de fallecimiento del paciente. Véase, entre otras muchas sentencias de esta sección y Sala, la de fecha 13-9-2012, n. ${ }^{\circ}$ 605/2012, que indemnizó en 18.000 euros el fallecimiento de un paciente oncológico; o la sentencia de fecha 07-02-2013, n. ${ }^{\circ} 119 / 2013$, en la que se indemnizó un fallecimiento con 12.000 euros, y con 8.000 euros en la sentencia de 
03-10-2012, n. ${ }^{\circ}$ 647/2012, por el fallecimiento de un paciente por cáncer de esófago.

En el segundo criterio, las resoluciones indemnizan íntegramente el daño, sin reducción de porcentajes, bien porque no consideran apropiado valorar conjeturas o bien porque consideran que la responsabilidad debe ser de quien ha infringido el deber objetivo de cuidado y ha creado el riesgo.

En el tercer criterio se aplica una regla de responsabilidad proporcional que, además, no deja de ser algo hipotético y pleno de incertidumbre causal. De hecho, a pesar de que la estadística hable a favor de un porcentaje de supervivencia del $80 \%$, el paciente podría haber tenido todas las posibilidades, pues no deja de ser nada más que una estadística. Además, conceder ese mismo porcentaje de indemnización podría resultar injusto, pues existe mayor probabilidad de que se encontrara en ese $80 \%$ de los que superan la enfermedad $y$, en tal supuesto, recuperar la salud si hubiera sido sometido al tratamiento adecuado con un $100 \%$ de posibilidades (todos los que se encuentran en el $80 \%$ superarían la enfermedad).

La Sala Tercera del Tribunal Supremo, sin mencionar el criterio de la pérdida de oportunidad y con aplicación de otra doctrina, del principio de facilidad probatoria al que hace referencia el artículo 217 de la Ley de Enjuiciamiento Civil, resuelve este conflicto de una manera absolutamente diferente y llena de lógica al advertir que, demostrada la mala praxis y siempre que exista tratamiento para la patología, se debe desplazar la carga de la prueba a la Administración pública para que sea esta la que acredite qué daños y en qué porcentaje se habrían evitado u ocasionado, en su caso, pues no se puede trasladar a la víctima la carga de la prueba de un hecho de demostración imposible. Así se pronuncia el Tribunal Supremo Sala 3. $\underline{a}$, sec. 4. $\stackrel{a}{\text {, en }}$ Sentencia de 26-3-2012, y sec. 6. , sentencias de 7-7-2008, 20-03-2007 y 17-07-2007.

Incluso, podemos añadir que, en algunos supuestos oncológicos, por ejemplo, en los que únicamente existiera un tratamiento paliativo, si este se aplica tardíamente es lógico concluir que el paciente fallecería de todas formas, pero podríamos plantear la indemnización dado que, quizá, habría fallecido más tarde con un tratamiento iniciado de forma precoz. Todos falleceremos, eso sí es seguro, la cuestión es cuándo, y si ese momento llega antes de lo esperado el fallecimiento traerá causa en la infracción del deber de cuidado.

Por otro lado, la descripción de las sentencias dictadas en relación con neoplasias malignas y patologías neurológicas a lo largo de 12 años nos muestra principalmente que estos procesos son de «alto riesgo» judicial en cuanto a la pérdida de la oportunidad asistencial se refiere (más de cuatro de cada 10 sentencias por pérdida de oportunidad asistencial en el ámbito contencioso-administrativo, dictadas en España, está relacionada con patología oncológica o neurológica). Esto indica que en ambas materias es donde más frecuentemente se aplica la teoría de la pérdida de oportunidad, precisamente porque en este terreno se hace sumamente difícil establecer la relación de causalidad con certeza.

El error diagnóstico fue el hecho imputado motivo de la sentencia $(66,5 \%$ de los casos analizados) más frecuente. Dicho error es también recogido en otras series, como el motivo más frecuente de reclamación. Así, por ejemplo, en un reciente estudio sueco, se recogieron las reclamaciones judiciales de 134 mujeres (desde 1995 hasta 2006) que habían presentado los efectos adversos de un diagnóstico tardío de cáncer de mama ${ }^{12}$. El diagnóstico erróneo fue causado por un examen clínico o radiológico incompleto y/o por una deficiente interpretación de dichos exámenes ${ }^{12}$. Se consideró que, al menos, en uno de cada 4 casos, el retraso diagnóstico conllevó un impacto negativo en la evolución de la paciente ${ }^{12}$. En otra revisión de 559 casos de quejas por negligencia en patologías neurológicas ocurridos en el Sistema Nacional de Salud del Reino Unido, entre los años 1995 y 2005 , el error diagnóstico se citó un $44 \%$ de las veces, siendo la enfermedad discal degenerativa (27\%), los tumores del sistema nervioso central (21\%), las infecciones del sistema nervioso central (11\%) y las hemorragias subaracnoideas $(9 \%)$ las patologías más reclamadas ${ }^{13}$. Finalmente, en un estudio más reciente del Sistema Nacional de Salud del Reino Unido, pero hasta el año $2012^{14}$, el error diagnóstico seguía siendo la causa más frecuente de reclamación, pero, a diferencia, del anterior, las patologías habían cambiado, siendo la patología medular, la enfermedad cerebrovascular, los tumores intracraneales, la hidrocefalia y la patología neuromuscular las más frecuentes ${ }^{14}$.

En conclusión, las sentencias dictadas por pérdida de oportunidad asistencial en el ámbito de la medicina pública española se han incrementado considerablemente estos últimos años, fundamentalmente por error diagnóstico y tratamiento inadecuado. En general, la mayoría de los pacientes fueron varones, que fallecieron, siendo la patología oncológica maligna y las enfermedades neurológicas las que más frecuentemente originaron la condena, cuya cuantía fue determinada en la mayor parte de las ocasiones a tanto alzado, sin atender a criterios determinados.

\section{Conflicto de intereses}

Los autores declaran no tener ningún conflicto de interés.

\section{Bibliografía}

1. Asúa González Cl. Pérdida de oportunidad en la responsabilidad sanitaria. Cizur Menor (Navarra): Thomson-Aranzadi; 2008.

2. Gallardo Castillo MJ. La responsabilidad patrimonial de la administración sanitaria. Barcelona: Bosch; 2009.

3. Sardinero García C. Responsabilidad administrativa, civil y penal por falta de información en el ámbito clínico: criterios indemnizatorios. Valencia: Ed. Tirant Lo Blanch; 2016.

4. Saigí Ullastre U, Gómez Durán EL, Arimany Manso J. Cuantificación de la pérdida de oportunidad en responsabilidad profesional médica. Rev Esp Med Legal. 2013;39:157-61.

5. Gómez-Durán EL, Mulà-Rosías JA, Lailla-Vicens JM, Benet-Travé J, Arimany-Manso J. Analysis of obstetrics and gynecology professional liability claims in Catalonia, Spain (1986-2010). J Forensic Leg Med. 2013;20:442-6.

6. Martín-Casals M, Ribot Igualada J, Sole Feliu J. Medical malpractice liability in Spain: Cases, trends and developments. Eur J Health Law. 2003;10:153-81.

7. Santiago-Sáez A, Perea-Pérez B, Albarrán-Juan ME, LabajoGonzález E, Anadón-Baselga MJ, Almendral-Garrote J. Análisis de las sentencias judiciales en la práctica de la cardiología resueltas en segunda instancia en España entre 1992 y 2007. Rev Esp Cardiol (Engl Ed). 2012;65:801-6.

8. Santiago-Sáez A, Perea-Perez B, Albarrán-Juan ME, LabajoGonzález E, Reneses-Prieto B. Análisis de sentencias judiciales 
contra psiquiatras resueltas en segunda instancia en España entre 1992 y 2007. Actas Esp Psiquiatr. 2011;39:393-400.

9. Santiago-Sáez A, Perea-Perez B, Labajo-González E, AlbarránJuan ME, Barcia JA. Análisis de sentencias judiciales contra neurocirujanos resueltas en segunda instancia en España entre 1995 y 2007. Neurocirugia (Astur). 2010;21:53-60.

10. Cardoso-Cita Z, Perea-Pérez B, Albarrán-Juan ME, LabajoGonzález ME, López-Durán L, Marco-Martínez F, et al. Análisis de sentencias judiciales relativas a negligencias médicas emitidas contra traumatólogos entre 1995 y 2011. Rev Esp Cir Ortop Traumatol. 2016;60:29-37.
11. Giraldo P, Sato L, Sala M, Comas M, Dywer K, Castells X. A retrospective review of medical errors adjudicated in court between 2002 and 2012 in Spain. Int J Qual Health Care. 2016;28:33-9.

12. Hafstrom L, Johansson H, Ahlberg J. Diagnostic delay of breast cancer - an analysis of claims to Swedish Board of Malpractice (LOF). Breast. 2011;20:539-42.

13. McNeill A. Neurological negligence claims in the NHS from 1995 to 2005. Eur J Neurol. 2007;14:399-402.

14. Coysh T, Breen DP. A nationwide analysis of successful litigation claims in neurological practice. JRSM Open. 2014;5, 2042533313518914. 